\title{
Visiting Student Away Rotations in Ophthalmology: A Study of Medical Students' Experiences and Perspectives
}

\author{
Sunny B. Patel, MD ${ }^{1}$ Lisa D. Kelly, MD² \\ ${ }^{1}$ Department of Ophthalmology, Rush University Medical Center, \\ Chicago, Illinois \\ 2 Department of Ophthalmology, University of Cincinnati, \\ Cincinnati, Ohio
}

\author{
Address for correspondence Lisa D. Kelly, MD, Department of \\ Ophthalmology, University of Cincinnati College of Medicine, Building \\ 5111, 231 Albert Sabin Way, Cincinnati, OH 45267-0527 \\ (e-mail: lisa.kelly@uc.edu).
}

J Acad Ophthalmol 2020;12:e52-e56.

\begin{abstract}
Keywords

- audition

- away rotation

- education

- elective

- match

- medical student

- ophthalmology

- residency

- visiting student

Background Despite the frequency of medical students' participation in ophthalmology clerkships away from their home institution, the impact and benefit of these clerkships have remained uninvestigated. To date, no study has focused specifically on medical student perspectives of away ophthalmology clerkships.

Objective The purpose of the study was to evaluate the medical students' perspectives on and experience with away rotations in ophthalmology, and assess their effect on residency Match outcomes.

Methods An anonymous, original, online survey was designed and distributed to applicants of the 2015 to 2018 ophthalmology Match cycles.

Results A total of 69 responses from nine medical institutions were collected $(62 \%$ response rate). Forty-one respondents (59\%) chose to perform at least one away rotation. Among away rotators, the mean number performed was 1.44. Thirty-seven away rotators $(90 \%)$ reported receiving an interview from at least one host institution they visited. The average estimated cost of an away rotation was $\sim 1,709$ U.S. dollars. With a $95.7 \%$ overall match rate among the respondents, no statistically significant difference was seen in match rates between away rotators and nonaway rotators $(p=0.564)$. Among the away rotators, the mean position on their rank order list matched was 2.34 , while the nonaway rotators matched at a mean position of 2.13 $(p=0.383)$.

Conclusion No association between away participation and success in the San Francisco Match was observed in this study. However, study participants did experience non-Match-related benefits from away elective participation.
\end{abstract}

Each fall, $\sim 600$ medical students and graduates apply to Accreditation Council for Graduate Medical Education accredited ophthalmology residency programs through the San Francisco (SF) Residency and Fellowship Matching Services, also known as the SF Match. Most applicants seek to design a robust application and include elements that enhance their likelihood of success in the Match process. An element, often

received

July 14, 2019

accepted after revision

March 1, 2020
DOI https://doi.org/

10.1055/s-0040-1709176. ISSN 2475-4757. considered is enrolling in a visiting student elective, also known as an away, audition, or extramural rotation. The "away" is simply defined as a clinical rotation performed outside of a student's home institution. Ophthalmology Match applicants must decide the value of pursuing an away rotation and advisors have limited evidence-based guidance to offer applicants.
Copyright $\odot 2020$ by Thieme Medical Publishers, Inc., 333 Seventh Avenue, New York, NY 10001, USA. Tel: +1(212) 760-0888.
License terms

(ㅇ)(1) $\Theta \circledast$ 
Proposed reasons in support of performing a visiting elective include improved chances of one's matching, greater insight to a particular residency program, increased knowledge about the specialty, and an offer of networking opportunities with a broader range of ophthalmologists.

However, participation in an away rotation is costly, stressful, and consumes valuable curriculum time. In fact, in regard to visiting rotations among all fields, the average expenditure per rotation observed in the literature was cited between 958 and 2,494 U.S. dollars. ${ }^{1,2}$ There is also an opportunity cost. Away electives are typically performed during the fourth year of medical school and compete with curriculum time that might otherwise be used for broadbased medical coursework in other medical specialties. Finally, a potential applicant might decrease their probability of matching at a program if faculty members find the away elective student's performance unimpressive.

In many nonophthalmology specialties, the decision of whether to participate in an away elective is unambiguous. For example, studies have shown in specialties such as orthopaedic surgery, neurosurgery, urology, and radiation oncology, the benefits outweigh the risks with respect to matching in the various specialties. ${ }^{3-6}$ On the contrary, in specialties such as internal medicine, general surgery, and family medicine, visiting student rotations have not proven to have a beneficial effect. ${ }^{3,7,8}$ In ophthalmology, however, no consensus about away rotations has been reached. ${ }^{9,10}$ Lee et al noted the audition elective had likely little to no benefit of improving one's chance of matching at a respective program despite widespread belief. ${ }^{10}$ Furthermore, in a 2009 survey of 46 residency program directors, audition electives were ranked 12th in regard to importance, among a group of 16 residency selection criteria. ${ }^{11}$ Nonetheless, the prevalence of ophthalmology bound students performing away rotations ranges as high as 70 to $90 \% .^{1,2}$

This ultimately leaves physicians, program and education directors, and mentors of these medical students in a difficult position from an academic advising standpoint. There are currently two major gaps in the body of knowledge on the subject. In the literature regarding away rotations, no study to date focused specifically on ophthalmology. Second, studies have seldom reported on away electives from the viewpoint of the medical student. Therefore, the objective of our study is twofold: to evaluate the medical student perspective on and experience with away clerkships in ophthalmology, and assess the effect of these away rotations on residency Match outcomes.

\section{Methods}

To accomplish these objectives, a 19-question, anonymous, skip logic, original survey was designed and distributed to former ophthalmology Match applicants, with approval from the Institutional Review Board of the University of Cincinnati. The questionnaire included qualitative and quantitative domains. Questions aimed to investigate information related to away rotation experience(s), potential effects on Match outcomes, motivations behind participat- ing in or abstaining from an away rotation, demographics, and academic performance.

The survey included closed-ended responses (e.g., multiple-choice questions and Likert scales) as well as open-ended free-text responses. Any ophthalmology Match applicant of the 2015 to 2018 SF Match cycles was eligible to participate. Participants were not excluded based on their success or failure of matching into ophthalmology, nor their participation in or abstinence from a visiting student elective. The online questionnaire was administered through SurveyMonkey and sent to potential participants via e-mail.

In effort to maximize response rate, increase willingness to give a candid response, and include applicants who did not match, participants were recruited directly from their current or graduated medical schools. All medical institutions associated with the Association of University Professors of Ophthalmology e-mail Listserv were invited to take part in the study. The medical student education directors of ophthalmology of institutions who expressed interest were then asked to contact their respective applicants of the 2015 to 2018 ophthalmology Match cycles and provide the online questionnaire via e-mail. Eligible participants were given 1 month to complete the survey. Data were consolidated and statistical analysis performed with Microsoft Excel 2017, Microsoft Corporation. Analyses (e.g., Fisher's exact test, Student's unpaired $t$-test, Pearson's Chi-square analysis with Yates correction) were performed to assess the effect of away rotations on Match outcomes.

\section{Results}

Nine institutions agreed to take part in the study and a total of 69 responses were collected during the study period, with a $62 \%$ response rate. The four Match cycles in which participants applied were evenly represented (-Fig. 1). The medical schools were predominantly in the Midwest, with no institutions from the western coast of the United States participating (-Fig. 2). Of the 69 participants, 54\% were female, 30\% reported Alpha Omega Alpha status, and 64\%

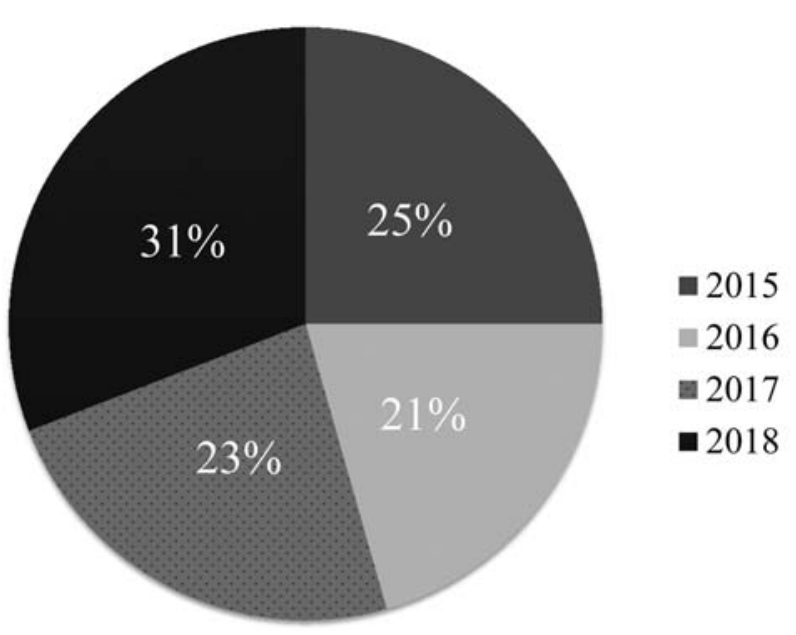

Fig. 1 Match cycle years applied by survey participants. 


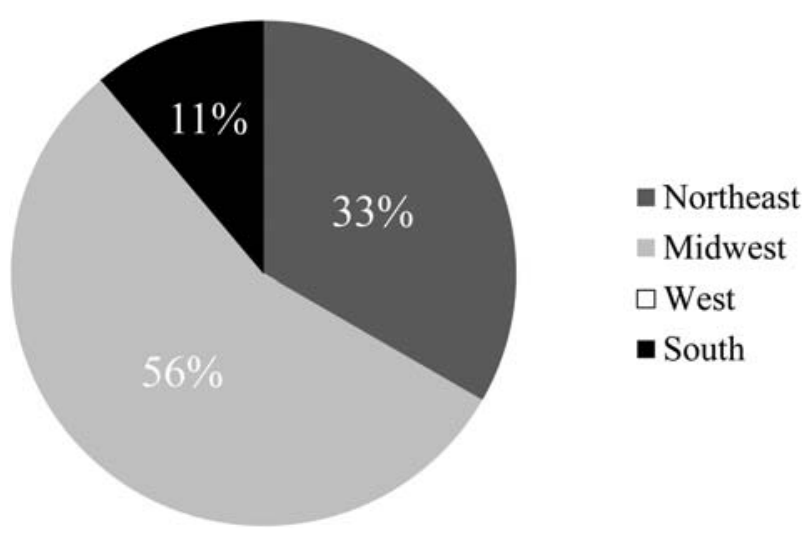

Fig. 2 Geographic distribution of participating medical institutions.

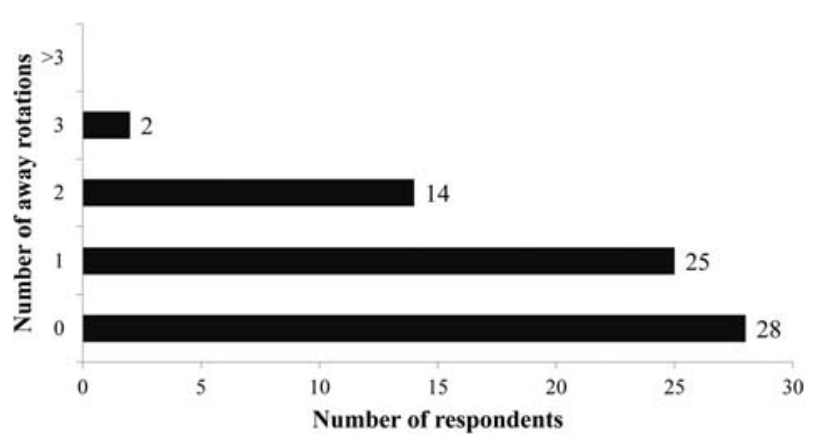

Fig. 3 Number of away rotations performed by survey participants.

scored at or above the average United States Medical Licensing Examination Step 1 score for matched applicants.

-Figure 3 demonstrated the number of rotations the cohort performed. Forty-two percent of respondents did not participate in an away rotation, while $58 \%$ performed at least one. The overall mean number of visiting electives performed per applicant was 0.86 . However, among away rotators, the mean number performed was 1.44 . In evaluating primary motivations, $43 \%$ of nonaway rotators stated the primary reason for electing not to perform an away was because they did not believe it would improve Match outcomes. Of note, one respondent reported abstaining due to cost and two reported not receiving an away rotation despite applying. Meanwhile, the away rotators stated the primary reason for electing to perform an away was to audition at a specific program (39\%), improve chances of matching in a geographic area (29\%), and increase overall Match competitiveness (17\%). The primary reason for choosing the specific host institution was geographic preference (48\%), followed by the pedigree or reputation of the program (29\%).

In terms of the greatest benefit of performing an away rotation, 39\% of former applicants reported it helped gain insight into the respective residency program and 29\% believed it improved Match outcomes. While no respondents listed educational purpose as their primary motivation in participating in an away, $22 \%$ of respondents felt this was the greatest benefit. The difficulty in trying to impress physician teams was most commonly reported as the greatest challenge, followed by learning a new hospital system and cost. Respondents were asked to estimate the expense of a single away rotation, including room, board, transportation, etc. The average estimated cost of an away rotation was $\sim 1,709 \mathrm{U}$. S. dollars ranging between 200 to 3,500 U.S. dollars (-Fig. 4). Twenty-two percent of participants were unable to estimate the cost.

Only $17 \%$ of respondents who performed an away reported obtaining a letter of recommendation during the rotation. Ninety percent of participants reported receiving an interview from at least one host institution at which they performed an away elective. In assessing how the away rotation affected one's rank list, $62 \%$ of respondents reported ranking at least one host institution they visited higher, due to their experience.

Away rotators were asked to what extent they either agreed or disagreed with the following statement: "I feel my away rotation improved my chances of matching." Sixty percent of away rotators agreed or strongly agreed with the sentiment. When asked whether applicants would recommend future medical students participate in an away rotation, those who did not perform an away were largely ambivalent: $83 \%$ neither agreeing nor disagreeing with the recommendation. Meanwhile, among those who performed an away rotation, $85 \%$ of respondents either agreed or strongly agreed.

In evaluating Match outcomes, only three former applicants in our sample did not match, providing a $95.7 \%$ overall match rate. - Table 1 illustrates the baseline characteristics and Match outcomes between the away rotators and nonaway rotators. Of the 41 respondents who performed a visiting rotation, one did not match. Of the 28 who did not perform an away elective, two

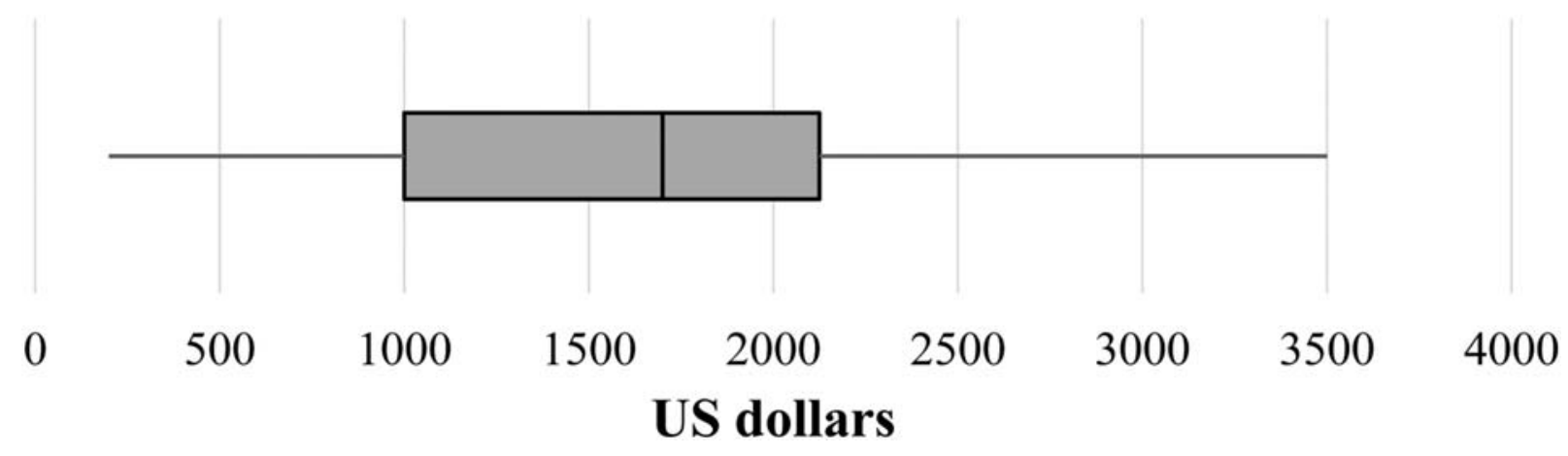

Fig. 4 Box plot demonstrating the estimated average cost of a single away rotation. 
Table 1 Baseline characteristics and Match outcomes of away rotators versus nonaway rotators

\begin{tabular}{|l|l|l|l|}
\hline & $\begin{array}{l}\text { Away } \\
\text { rotators }\end{array}$ & $\begin{array}{l}\text { Nonaway } \\
\text { rotators }\end{array}$ & $p$-Value \\
\hline Total & 41 & 28 & $\mathrm{~N} / \mathrm{A}$ \\
\hline $\begin{array}{l}\%>\text { USMLE step }_{\text {mean }^{\mathrm{a}}} \\
\% \text { AOA status }\end{array}$ & 34.54 & 71.43 & $\mathrm{~N} / \mathrm{A}$ \\
\hline Matched (\%) & $40(97.5)$ & $26(92.9)$ & $0.564^{\mathrm{b}}$ \\
\hline $\begin{array}{l}\text { Mean RoL position } \\
\text { matched (95\% CI) }\end{array}$ & $2.34(0.53)$ & $2.13(0.56)$ & $0.383^{\mathrm{c}}$ \\
\hline $\begin{array}{l}\text { Median RoL } \\
\text { position matched }\end{array}$ & 2 & 2 & $\mathrm{~N} / \mathrm{A}$ \\
\hline $\begin{array}{l}\text { Matched first } \\
\text { choice (\%) }\end{array}$ & $18(47.37)$ & $11(47.83)$ & $0.818^{\mathrm{d}}$ \\
\hline $\begin{array}{l}\text { Matched top } \\
3 \text { choices (\%) }\end{array}$ & $30(78.95)$ & $19(82.61)$ & $0.987^{\mathrm{d}}$ \\
\hline
\end{tabular}

Abbreviations: AOA, Alpha Omega Alpha; $\mathrm{Cl}$, confidence interval; N/A, not available; RoL, rank order list; USMLE, United States Medical Licensing Examination.

${ }^{a}$ Annual mean for matched applicants.

bFisher's exact test.

'Student's unpaired t-test.

'Pearson's chi-square analysis with Yates correction.

former students did not match $(p=0.564)$. The study investigated how high applicants matched on their rank order lists (RoL) to serve as a surrogate for how well they matched. Among away rotators, the mean position on their matched RoL was 2.34 , while the nonaway rotators matched at a mean position of $2.13(p=0.383)$. Approximately $47.4 \%$ of away rotators matched at their first choice program, while $47.8 \%$ of nonaway rotators matched at their first choice $(p=0.818)$. About $79 \%$ of away rotators matched within the top three choices of their rank list, while $82.6 \%$ of nonaway rotators matched within their top 3 choices $(p=0.987)$.

\section{Discussion}

Each year, medical students are tasked with the difficult decision of whether to participate in a visiting student elective. While the choice is much more straightforward in many other medical specialties, the correct decision for prospective ophthalmology applicants is far less apparent. ${ }^{9,10}$ Our study showed a sample of 69 former ophthalmology residency applicants, $58 \%$ of who chose to perform an away rotation. This may demonstrate a decline in the emphasis on their importance, as previous literature reports a prevalence ranging between 70 and $90 \%{ }^{1,2}$ It may also reflect regional variation given the majority of respondents in this study attended a medical institution in the Midwestern region of the United States (-Fig. 2). Among those who performed at least one, the average number of rotations per applicant was between one and two electives.

There are several challenges associated with participating in an away based on our study. Students can expect a steep learning curve associated with unfamiliar physicians' teams and hospital systems. There is also a significant financial cost. Our study found a cost of $\sim 1,700$ U.S. dollars per rotation, which is in the range of what has been observed in the literature for other specialties. ${ }^{1,2}$ Keep in mind that the away elective expense is in addition to the costs of the ophthalmology residency application process. Such high costs also present a challenge for residency programs, as they risk inequitably selecting for applicants with greater financial resources. Finally, due to the early timeline of the ophthalmology Match, students cannot reliably expect to obtain a letter of recommendation as a benefit of their away experience, as may be expected in other specialties.

Our study results revealed that students obtained an interview from at least one host institution they visited, as well as gained insight into that program. So, although a reasonable expectation, receiving an interview invitation is certainly not guaranteed. A survey of 65 ophthalmology resident selection committee representatives reported only about half routinely interview applicants who audition. ${ }^{12}$ Lee et al also importantly questioned the significance of the interview, if only provided as a courtesy for visiting as a rotator. ${ }^{10}$

Improving performance in the Match was the primary motivation of students who did away rotations; however, learning more about a specific program was the greatest benefit most commonly reported. Regarding the recommendation of whether future students should participate in an away rotation, the majority of those who performed an away elective agreed with the recommendation. Meanwhile, the majority of nonaway rotators were neither for nor against the recommendation.

With respect to the impact of away rotations on Match outcomes, based on our cohort, an away rotation experience did not alter the risk of an applicant failing to match. A caveat, however, is that only a small portion of applicants in this study failed to match. This was not representative of the percentage of applicants who failed to match during these years as compared with SF Match data. In further assessing if an away rotation affected how well an applicant matched (i. e., how high applicants matched on their RoL), no association between away participation and Match outcome was seen. We would recommend advisors make future applicants aware of this, but also inform them of the other potential benefits of performing a visiting elective discussed earlier.

Limitations of the study included potential selection bias, as institutions and applicants more successful with the Match might have been more likely to participate. This might explain the under-representation of applicants who did not match. Inherent limitations of an online survey included self-reported data subject to recall bias and the inability to monitor an applicant's interpretation of a question. The sample size limited the power of our statistical analysis. However, the study still provided the largest sample size of ophthalmology applicants reported to date in regard to away rotations. For future investigations, an important inquiry would be whether an away elective increased the likelihood of matching at the host institution. While an important question going forward, it was not the purpose of this study. 


\section{Conclusion}

The study is a first of its kind in regard to what has previously been examined on away rotations in ophthalmology. It is an initial step in learning more about an important area that has not otherwise been well investigated. We hope the information collected may serve as a resource in guiding future students interested in ophthalmology with their decisions concerning performing away rotations-a decision that is ultimately multifactorial and based on a combination of a student's motivations, expectations, prior experiences, and remaining application.

\section{Conflict of Interest}

None declared.

\section{Acknowledgments}

The authors would like to thank the following participating institutions for their assistance in the recruitment of eligible participants for the study: Cleveland Clinic Lerner College of Medicine, McGovern Medical School at the University of Texas Health Science Center at Houston, Northwestern University Feinberg School of Medicine, The Ohio State University College of Medicine, Tufts University School of Medicine, University of Maryland School of Medicine, University of Michigan Medical School, University of Cincinnati College of Medicine, and Yale School of Medicine.

\section{References}

1 Oladeji LO, Raley JA, Smith S, Perez JL, McGwin G, Ponce BA. Behind the Match process: is there any financial difference lurking below the specialty of choice? Am Surg 2016;82(12): 1163-1168

2 Winterton M, Ahn J, Bernstein J. The prevalence and cost of medical student visiting rotations. BMC Med Educ 2016;16(01): 291

3 Higgins E, Newman L, Halligan K, Miller M, Schwab S, Kosowicz L. Do audition electives impact match success? Med Educ Online 2016;21:31325

4 Weissbart SJ, Stock JA, Wein AJ. Program directors' criteria for selection into urology residency. Urology 2015;85(04): 731-736

5 Camp CL, Sousa PL, Hanssen AD, et al. The cost of getting into orthopedic residency: analysis of applicant demographics, expenditures, and the value of away rotations. J Surg Educ 2016;73(05):886-891

6 Al Khalili K, Chalouhi N, Tjoumakaris S, et al. Programs selection criteria for neurological surgery applicants in the United States: a national survey for neurological surgery program directors. World Neurosurg 2014;81(3-4):473-477.e2

7 Fabri PJ, Powell DL, Cupps NB. Is there value in audition extramurals? Am J Surg 1995;169(03):338-340

8 Vogt HB, Thanel FH, Hearns VL. The audition elective and its relation to success in the National Resident Matching Program. Teach Learn Med 2000;12(02):78-80

9 Grubbs JR Jr, Mian SI. Advising students interested in ophthalmology: a summary of the evidence. Ophthalmology 2016;123 (07):1406-1410

10 Lee AG, Golnik KC, Oetting TA, et al. Re-engineering the resident applicant selection process in ophthalmology: a literature review and recommendations for improvement. Surv Ophthalmol 2008; 53(02):164-176

11 Green M, Jones P, Thomas JX Jr. Selection criteria for residency: results of a national program directors survey. Acad Med 2009;84 (03):362-367

12 Nallasamy S, Uhler T, Nallasamy N, Tapino PJ, Volpe NJ. Ophthalmology resident selection: current trends in selection criteria and improving the process. Ophthalmology 2010;117 (05):1041-1047 